\author{
Reactor and Nuclear Systems Division
}

\title{
IMPLEMENT METHOD FOR AUTOMATED TESTING OF MARKOV CHAIN CONVERGENCE INTO INVERSE FOR ORNL12-RS-108J: ADVANCED MULTI-DIMENSIONAL FORWARD AND INVERSE MODELING
}

\author{
Keith C. Bledsoe \\ Oak Ridge National Laboratory \\ P.O. Box 2008 \\ Bldg. 5700, MS-6170 \\ Oak Ridge, TN 37831-6170
}

Date published: April 2015

\author{
Prepared for the \\ Technology Integration Program (TI) \\ Office of Emergency Response (NA-42) \\ National Nuclear Security Administration (NNSA) \\ U.S. Department of Energy (US DOE)
}

Prepared by

OAK RIDGE NATIONAL LABORATORY

Oak Ridge, Tennessee 37831-6170

managed by

UT-BATTELLE, LLC

for the

US Department of Energy

under contract DE-AC05-00OR22725 


\title{
DOCUMENT AVAILABILITY
}

Reports produced after January 1, 1996, are generally available free via US Department of Energy (DOE) SciTech Connect.

Website http://www.osti.gov/scitech/

Reports produced before January 1, 1996, may be purchased by members of the public from the following source:

\author{
National Technical Information Service \\ 5285 Port Royal Road \\ Springfield, VA 22161 \\ Telephone 703-605-6000 (1-800-553-6847) \\ TDD 703-487-4639 \\ Fax 703-605-6900 \\ E-mail info@ntis.gov \\ Website http://www.ntis.gov/help/ordermethods.aspx
}

Reports are available to DOE employees, DOE contractors, Energy Technology Data Exchange representatives, and International Nuclear Information System representatives from the following source:

Office of Scientific and Technical Information

PO Box 62

Oak Ridge, TN 37831

Telephone 865-576-8401

Fax 865-576-5728

E-mail reports@osti.gov

Website http://www.osti.gov/contact.html

This report was prepared as an account of work sponsored by an agency of the United States Government. Neither the United States Government nor any agency thereof, nor any of their employees, makes any warranty, express or implied, or assumes any legal liability or responsibility for the accuracy, completeness, or usefulness of any information, apparatus, product, or process disclosed, or represents that its use would not infringe privately owned rights. Reference herein to any specific commercial product, process, or service by trade name, trademark, manufacturer, or otherwise, does not necessarily constitute or imply its endorsement, recommendation, or favoring by the United States Government or any agency thereof. The views and opinions of authors expressed herein do not necessarily state or reflect those of the United States Government or any agency thereof. 


\section{TABLE OF CONTENTS}

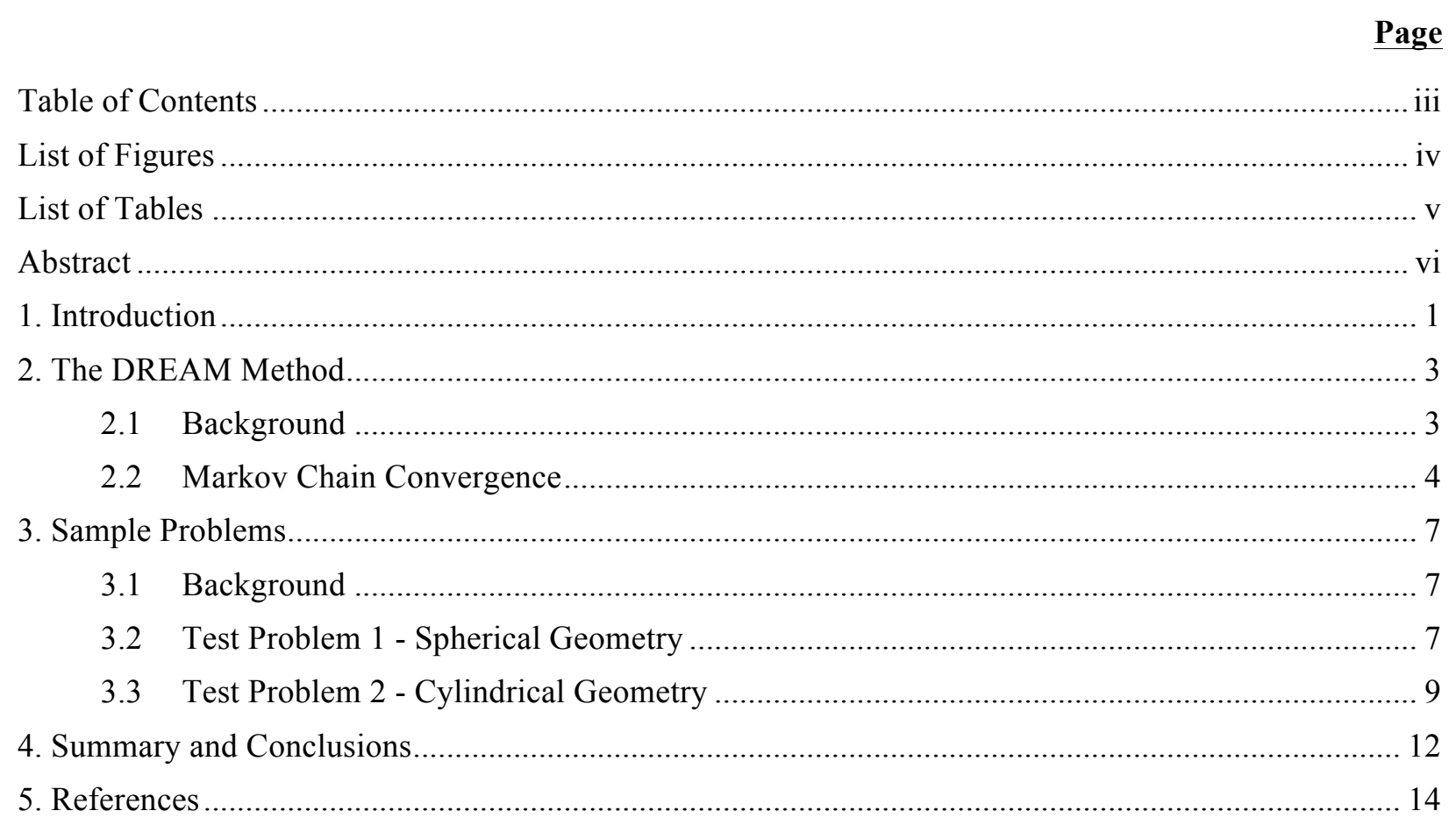




\section{LIST OF FIGURES}

Page

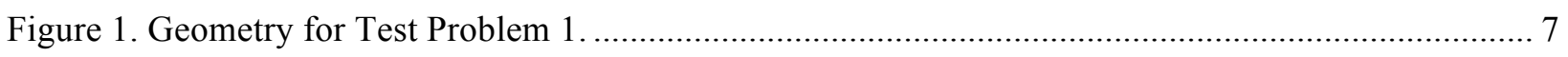

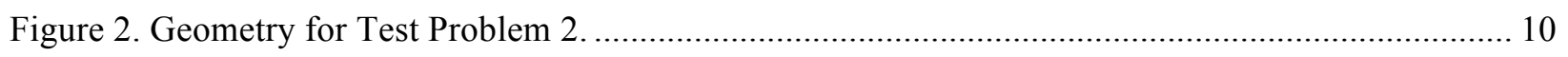




\section{LIST OF TABLES}

Page

Table 1. Initial guidelines for the number of generations to use with the DREAM method in INVERSE . 4

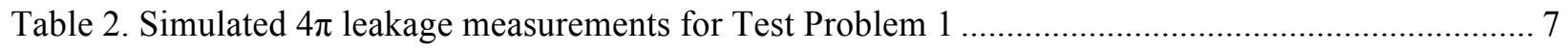

Table 3. Results of the Gelman-Rubin Method for Test Problem 1 ….................................................. 8

Table 4. Simulated scalar flux measurements for Test Problem 2 ........................................................ 11

Table 5. Results of the Gelman-Rubin Method for Test Problem 2 ....................................................... 11 


\begin{abstract}
The DiffeRential Evolution Adaptive Metropolis (DREAM) method is a powerful optimization/uncertainty quantification tool used to solve inverse transport problems in Los Alamos National Laboratory's INVERSE code system. The DREAM method has been shown to be adept at accurate uncertainty quantification, but it can be very computationally demanding. Previously, the DREAM method in INVERSE performed a user-defined number of particle transport calculations. This placed a burden on the user to guess the number of calculations that would be required to accurately solve any given problem. This report discusses a new approach that has been implemented into INVERSE, the Gelman-Rubin convergence metric. This metric automatically detects when an appropriate number of transport calculations have been completed and the uncertainty in the inverse problem has been accurately calculated. In a test problem with a spherical geometry, this method was found to decrease the number of transport calculations (and thus time required) to solve a problem by an average of over $90 \%$. In a cylindrical test geometry, a 75\% decrease was obtained.
\end{abstract}




\section{INTRODUCTION}

The forward problem of particle transport can be defined as "the determination of the population of particles in a system given the physical parameters of the system, including the particle source." Using this calculated particle population, values such as detector responses can be calculated. The inverse problem asks the opposite question: if detector responses are determined by measurement, can the physical parameters of a system be reconstructed?

Los Alamos National Laboratory has a software package called INVERSE that is capable of solving inverse transport problems of interest to the NA-42 mission space, including the problems of material interface location identification, source isotopic content identification, material density identification, and shield material identification. INVERSE uses a variety of methods for solving inverse transport problems. One of the most promising is the DiffeRential Evolution Adaptive Metropolis (DREAM) method, a hybridization of Markov chain Monte Carlo sampling and the evolutionary algorithm differential evolution [1]. The DREAM method has been shown to be an effective method for solving and quantifying uncertainty in inverse transport problems, but its use of many multiple forward simulations makes it computationally expensive.

One of the most glaring issues with the implementation of DREAM within INVERSE was the burden placed on the user to decide the number of transport calculations required to accurately solve the problem. The user could only follow very general guidelines in choosing this number, and it was unclear whether the user-determined value was too large, and would waste valuable time and computing resources, or if it may have been too small, not sufficiently solving the problem. In order to overcome this burden, the Gelman-Rubin convergence criterion [2] has been implemented in this work. The GelmanRubin method is a technique for detecting convergence of simultaneous Markov chains exploring the same solution space. It uses a measure of the variance of chain states within the individual chains and between the chains in order to make this detection. This method has proven useful for problems in fields such as uncertainty quantification for ground penetrating radar data [3] and groundwater modeling [4]. 


\section{THE DREAM METHOD}

\subsection{BACKGROUND}

Markov chain Monte Carlo methods provide a generalized methodology for inferring the posterior distribution of the unknown parameters in an inverse transport problem. This posterior distribution represents the ranges that the unknown values may take given the measured data and its associated uncertainty. In an idealized problem in which there is no uncertainty in measurements, the posterior distribution for each unknown parameter would be a single value.

The posterior probability distribution $p\left(\mathbf{u} \mid M_{o}\right)$ represents the probability of a model $\mathbf{u}$ (where $\mathbf{u}$ is a vector representing postulated values for the unknown parameters) given observed measurements $M_{O}$. This distribution is proportional to a likelihood function times a prior probability distribution. For this study, it was assumed that the prior distribution had an equal probability that the parameter lies somewhere within its constraints, and a probability of zero that the parameter lies outside of its constraints. The likelihood function, $p\left(M_{o} \mid \mathbf{u}\right)$, is the probability of measurements $M_{o}$ given parameters $\mathbf{u}$. The likelihood function was defined as

$$
p\left(M_{o} \mid \mathbf{u}\right)=\exp \left[-\frac{1}{2} \sum_{d=1}^{D}\left(\frac{M_{d}(\mathbf{u})-M_{d, o}}{\sigma_{d}}\right)^{2}\right]
$$

where $D$ is the total number of detector measurements, $M_{d}(\mathbf{u})$ is the calculated measurement at detector $d$ for postulated parameter set $\mathbf{u}, M_{d, o}$ is the observed measurement for detector $d$, and $\sigma_{d}$ is the uncertainty in the measurement for detector $d$. Since the prior probability distribution is equal to 1.0 in feasible regions of the search space, the posterior distribution is equal to the likelihood: $p\left(\mathbf{u} \mid M_{o}\right)=p\left(M_{o} \mid \mathbf{u}\right)$. The goal of the inverse problem is to find the regions for which $p\left(\mathbf{u} \mid M_{o}\right)$ is near its maximum.

In the traditional Markov chain Monte Carlo (MCMC) approach, a single Markov chain is employed. The chain begins at some initial parameter set $\mathbf{u}_{\mathrm{t}}$ for which the posterior $p\left(\mathbf{u}_{\mathrm{t}} \mid M_{o}\right)$ is calculated, and then a trial parameter set is created. The posterior $p\left(\mathbf{u}_{\mathrm{t}+1} \mid M_{o}\right)$ is calculated for this trial parameter set, and the trial parameter set is either accepted or rejected according to the Metropolis acceptance probability

$$
\alpha\left(\mathbf{u}_{\mathrm{t}}, \mathbf{u}_{\mathrm{t}+1}\right)=\min \left[\frac{p\left(\mathbf{u}_{\mathrm{t}+1} \mid M_{o}\right)}{p\left(\mathbf{u}_{\mathrm{t}} \mid M_{o}\right)}, 1\right] .
$$

In Eq. (2) we see that if the trial point has a posterior larger than the current chain state (i.e., parameters $\mathbf{u}_{\mathrm{t}+1}$ yield a closer match between calculated and observed measurements), then the acceptance probability is 1 , and the chain is moved to the trial state. If parameters $\mathbf{u}_{\mathrm{t}+1}$ do not lead to a closer match between calculated parameters, they are still accepted with a probability equal to $p\left(\mathbf{u}_{\mathrm{t}+1} \mid M_{o}\right) / p\left(\mathbf{u}_{\mathrm{t}} \mid M_{o}\right)$. The chain progresses in this way until it creates the full posterior distribution describing the probabilities for the values of the unknown parameters. In this report, the creation and testing of the new chain state $\mathbf{u}_{\mathrm{t}+1}$ is referred to as a "generation" of the MCMC algorithm. New generations of the chain are created until the posterior distribution has been accurately constructed. 
Traditional MCMC approaches have generally been inefficient because trial parameters are either too close to the current point, leading to a high acceptance rate but slow convergence to the posterior distribution, or they are too far from the current point, leading to a low acceptance rate. The issue of choosing an appropriate trial parameter has been explored for many years. One particularly successful method for solving this problem is the DiffeRential Evolution Adaptive Metropolis (DREAM) algorithm. This method employs simultaneous multiple Markov chains (generally 3-5), and it uses the Differential Evolution algorithm to generate trial points for each chain. Suppose we have a set of $M$ chains, each containing a postulated parameter set $\mathbf{u}_{m}(m=1, \ldots, M)$. For chain $m$, the differential evolution approach generates a trial point according to

$$
\mathbf{u}_{m, \text { trial }}=\mathbf{u}_{m}+\gamma\left(\mathbf{u}_{R 1}-\mathbf{u}_{R 2}\right)+\mathbf{e}
$$

In Eq. (3), $\mathbf{u}_{R 1}$ and $\mathbf{u}_{R 2}$ are the current states of two randomly selected chains that are different from chain $i$ and different from each other. The term $\gamma$ is a scalar parameter set equal to $2.38 / \sqrt{2 N}$, where $N$ is the number of unknown parameters in the problem. The term $\mathbf{e}$ is a small scalar value used to increase diversity in the trial chain values. The DREAM approach for generating trial values for Markov chains has been shown to be highly successful for solving difficult optimization problems in the presence of noise.

\subsection{MARKOV CHAIN CONVERGENCE}

One of the most pressing questions in Markov chain sampling is how many generations are required to build accurate posterior distributions? Previously, this number of generations was set by the user of INVERSE. This put a particularly difficult burden on the user, because numerical experience has indicated that the necessary number of generations varies greatly by problem. In an (unpublished) user's guide provided to LANL staff members, the following guidelines were given:

Table 1. Initial guidelines for the number of generations to use with the DREAM method in INVERSE

\begin{tabular}{|c|c|}
\hline Number of parameters & Number of generations \\
\hline 1 & $10,000-20000$ \\
\hline 2 & $10,000-30,000$ \\
\hline 3 & $20,000-50,000$ \\
\hline 4 & $50,000-400,000$ \\
\hline
\end{tabular}

Table 1 indicates the difficulty of assigning a maximum number of generations. For instance, for four unknown parameters, anywhere between 50,000 and 400,000 generations were thought necessary.

In order to overcome this ambiguity, a new method has been implemented into INVERSE to automatically detect when the Markov chains have sufficiently sampled the posterior distribution. This method, known as the Gelman-Rubin convergence metric, is described in Ref. [2]. The Gelman-Rubin metric monitors the standard deviations both within and between the parallel Markov chains run by the DREAM method in order to detect when chains have stabilized to the posterior distribution. The metric is described by the following equations. The between-chain variance, measuring the variance between the $3-5$ chains in the DREAM method for parameter $n$, given by 


$$
B_{n}=\frac{L}{M-1} \sum_{m=1}^{M}\left(\bar{u}_{n, m}-\bar{u}_{n}\right)^{2}, \quad \text { where } \bar{u}_{i, m}=\frac{1}{L} \sum_{l=1}^{L} u_{n, m}^{l}, \quad \bar{u}_{i}=\frac{1}{M} \sum_{m=1}^{M} \bar{u}_{n, m}
$$

where $M$ is the number of chains, $L$ is the length of the chains (number of generations), and $u_{n, m}^{l}$ is the mean value of parameter $n$ for chain $m$ at generation $l$. The within-chain variance for parameter $n$, which gives a measure of the variance within the chain over the $L$ generations, is given by

$$
W_{n}=\frac{1}{M} \sum_{m=1}^{M} s_{m}^{2}, \text { where } s_{m}^{2}=\frac{1}{L-1} \sum_{l=1}^{L}\left(u_{n, m}^{l}-\bar{u}_{n, m}\right)^{2} .
$$

The between-chain and within-chain variances are then combined to form what is called the posterior marginal variance for parameter $n, \widehat{V}_{n}$ :

$$
\widehat{V}_{n}=\frac{L-1}{L} W_{n}+\frac{M+1}{L M} B_{n}
$$

If the $M$ chains have reached the target distribution, the posterior variance estimate should be very close to the within-chain variance $W$. Therefore, the ratio $\widehat{V} / W$ should be close to 1 . The square root of $\widehat{V} / W$, called the potential scale reduction factor (PSRF), is then used to measure whether the target distribution has been reached and thus accurately quantified uncertainty in the unknown parameters. In this work, a value of $\mathrm{PSRF}<1.1$ was used to indicate success. 


\section{SAMPLE PROBLEMS}

\subsection{BACKGROUND}

The Gelman-Rubin convergence metric was applied to two numerical test cases, one in a spherically symmetric geometry, and one in a cylindrical geometry. Both cases had a highly enriched uranium source with a composition of $94.73 \%{ }^{235} \mathrm{U}$ and $5.27 \%{ }^{238} \mathrm{U}$.

\subsection{TEST PROBLEM 1 - SPHERICAL GEOMETRY}

In the spherical test problem, the source has a radius of $8.741 \mathrm{~cm}$ and is surrounded by a layer of void with a radius of $12.4 \mathrm{~cm}$. This is followed by a $0.5 \mathrm{~cm}$-thick layer of lead and a $0.3 \mathrm{~cm}$-thick layer of aluminum. This geometry is shown in Figure 1. The measured values in this problem are the total $(4 \pi)$ leakages of the four strongest photon emission lines of uranium: 144-, 186-, 766-, and 1001-keV. "Measured" leakage values were simulated using Monte Carlo N particle (MCNP) transport code with a small number of histories in order to simulate the uncertainty of real measurement. Table 2 shows the simulated measured leakages for the system.

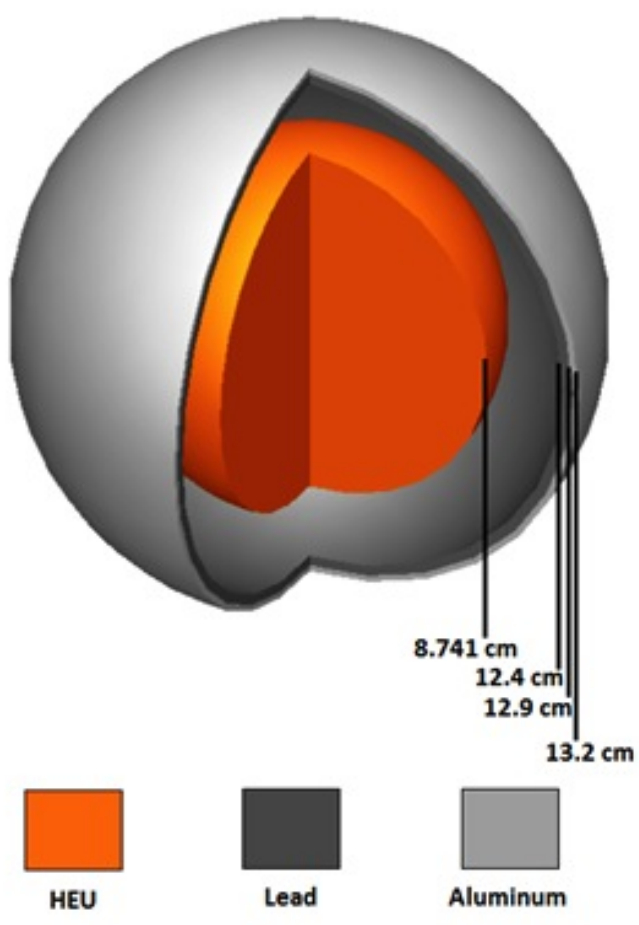

Figure 1. Geometry for Test Problem 1.

Table 2. Simulated $4 \pi$ leakage measurements for Test Problem 1 


\begin{tabular}{cc}
\hline Energy line (keV) & $\begin{array}{c}\text { Simulated } \\
\text { measurement }\end{array}$ \\
\hline 144 & $9.96 \times 10^{-1} \pm 0.56 \%$ \\
\hline 186 & $4.67 \times 10^{3} \pm 0.12 \%$ \\
\hline 766 & $2.53 \times 10^{3} \pm 4.26 \%$ \\
\hline 1001 & $9.86 \times 10^{3} \pm 2.32 \%$ \\
\hline
\end{tabular}

In this sample problem, the three interior radii $(8.741 \mathrm{~cm}, 12.4 \mathrm{~cm}$, and $12.9 \mathrm{~cm})$ in the problem are unknown. We assume that the only information we have about them is that they are within the $13.2 \mathrm{~cm}$ outer aluminum radius. Results for the DREAM method using the Gelman-Rubin metric and with a fixed total number of transport calculations following previous guidelines $(30,000$ generations, 150,000 total calculations) are presented in Table 3. In both cases, 10 independent DREAM calculations were conducted; each of these calculations used a different random number seed. Using the Gelman-Rubin diagnostic, an average of 12,622 calculations was required to accurately quantify uncertainty for the unknown radii. This is a $92 \%$ reduction in the number of transport calculations required (and thus time required) to solve the problem.

Table 3. Results of the Gelman-Rubin Method for Test Problem 1

\begin{tabular}{rcccc}
\hline \multicolumn{5}{c}{ DREAM with Gelman-Rubin Method } \\
\hline Test & Source radius & Inner lead radius & Outer lead radius & Transport calculations \\
\hline 1 & $8.58 \pm 0.21$ & $12.45 \pm 0.06$ & $12.96 \pm 0.07$ & 36,000 \\
2 & $8.56 \pm 0.26$ & $12.46 \pm 0.08$ & $12.96 \pm 0.08$ & 10,353 \\
3 & $8.54 \pm 0.22$ & $12.46 \pm 0.07$ & $12.97 \pm 0.07$ & 14,191 \\
4 & $8.56 \pm 0.26$ & $12.46 \pm 0.08$ & $12.96 \pm 0.08$ & 12,885 \\
5 & $8.56 \pm 0.31$ & $12.46 \pm 0.09$ & $12.96 \pm 0.10$ & 8,334 \\
6 & $8.58 \pm 0.26$ & $12.45 \pm 0.07$ & $12.95 \pm 0.07$ & 9,135 \\
7 & $8.58 \pm 0.26$ & $12.46 \pm 0.07$ & $12.96 \pm 0.07$ & 6,192 \\
8 & $8.53 \pm 0.24$ & $12.46 \pm 0.07$ & $12.96 \pm 0.07$ & 11,557 \\
9 & $8.56 \pm 0.25$ & $12.46 \pm 0.08$ & $12.96 \pm 0.08$ & 8,343 \\
10 & $8.59 \pm 0.30$ & $12.45 \pm 0.09$ & $12.95 \pm 0.09$ & \\
\hline & & DREAM without Gelman-Rubin Method & 150,000 \\
\hline Test & Source radius & Inner lead radius & Outer lead radius & Transport calculations \\
\hline 1 & $8.54 \pm 0.25$ & $12.46 \pm 0.07$ & $12.96 \pm 0.08$ & 150,000 \\
2 & $8.56 \pm 0.25$ & $12.46 \pm 0.07$ & $12.96 \pm 0.07$ & 150,000 \\
3 & $8.55 \pm 0.24$ & $12.46 \pm 0.07$ & $12.96 \pm 0.07$ & 150,000 \\
4 & $8.56 \pm 0.24$ & $12.46 \pm 0.07$ & $12.96 \pm 0.07$ & 150,000 \\
5 & $8.54 \pm 0.25$ & $12.47 \pm 0.07$ & $12.97 \pm 0.08$ & 150,000 \\
6 & $8.56 \pm 0.26$ & $12.46 \pm 0.07$ & $12.96 \pm 0.08$ & 150,000 \\
7 & $8.57 \pm 0.25$ & $12.46 \pm 0.07$ & $12.96 \pm 0.08$ & 150,000 \\
8 & $8.56 \pm 0.24$ & $12.46 \pm 0.07$ & $12.96 \pm 0.08$ & 150,000 \\
9 & $8.56 \pm 0.27$ & $12.46 \pm 0.08$ & $12.96 \pm 0.08$ & 150,000 \\
10 & $8.56 \pm 0.24$ & $12.46 \pm 0.07$ & $12.96 \pm 0.07$ & \\
\hline
\end{tabular}




\subsection{TEST PROBLEM 2 - CYLINDRICAL GEOMETRY}

The cylindrical test geometry is shown in Figure 2. The region of highly enriched uranium has a radius of $4.0 \mathrm{~cm}$. Its bottom is located at $1.5 \mathrm{~cm}$ and its top is at $6.0 \mathrm{~cm}$. The source is surrounded by layers of lead and aluminum shielding, and detectors below $(r, z)=(0.0 \mathrm{~cm},-1.0 \mathrm{~cm})$ and outside the radial face $(r, z)=$ $(10.0 \mathrm{~cm}, 4.0 \mathrm{~cm})$ of the geometry measure the uranium emission lines. The simulated measurements are given in 
Table 4.

Due to longer run times for two-dimensional problems, previous DREAM calculations were kept to a minimum of 90,000 transport calculations. A comparison of results obtained using the Gelman-Rubin convergence metric and the standard 90,000 transport calculations is given in Table 5. Using the GelmanRubin criterion, the average number of transport calculations required to accurately quantify the uncertainty on the unknown parameters was $22,875-$ a $75 \%$ decrease in the number of calculations required.
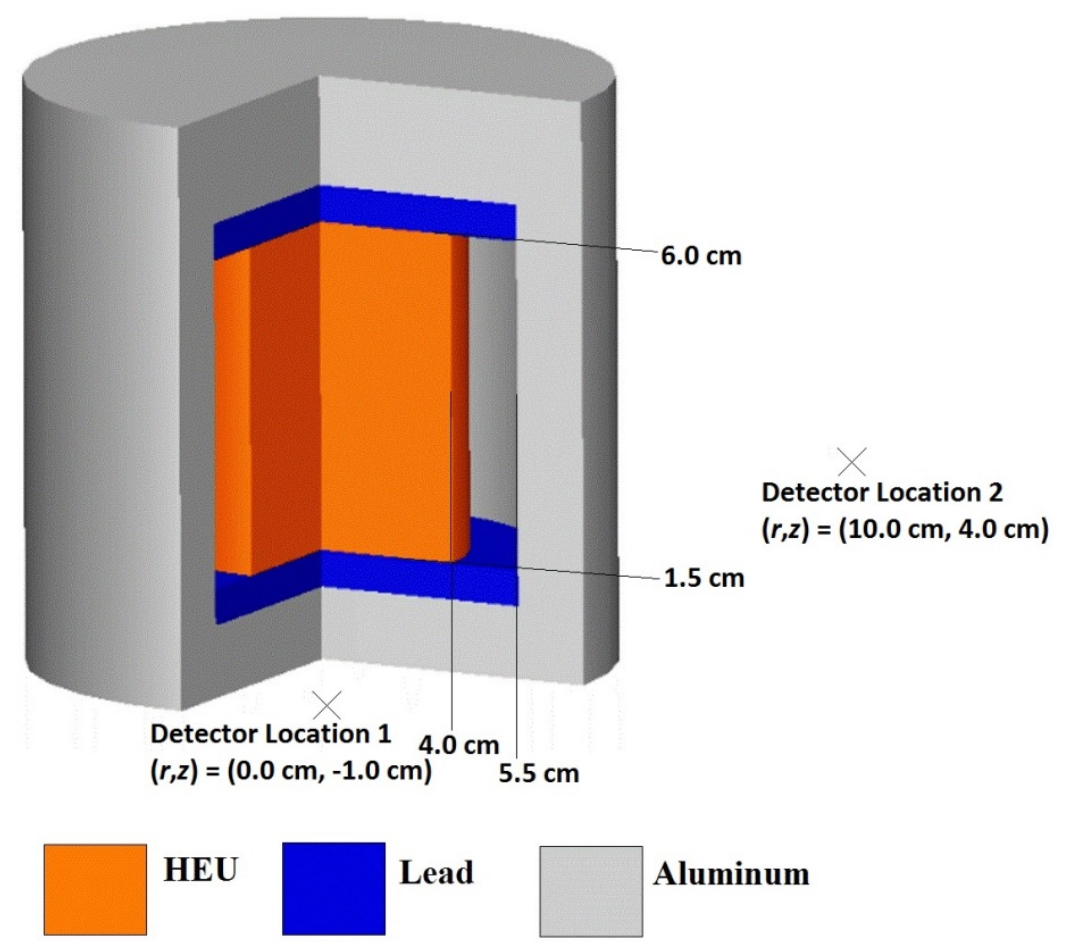

Figure 2. Geometry for Test Problem 2. 
Table 4. Simulated scalar flux measurements for Test Problem 2

\begin{tabular}{cc}
\hline Detector $\mathbf{1}(\boldsymbol{r}, \boldsymbol{z})$ & $\mathbf{( 0 . 0} \mathbf{c m}, \mathbf{- 1 . 0} \mathbf{c m})$ \\
\hline Energy line $(\mathrm{keV})$ & Simulated measurement \\
\hline 144 & $1.57 \times 10^{-3} \pm 60.45 \%$ \\
186 & $1.92 \times 10^{0} \pm 12.36 \%$ \\
766 & $1.69 \times 10^{0} \pm 1.77 \%$ \\
1001 & $7.10 \times 10^{0} \pm 1.49 \%$ \\
\hline Detector 2 (r,z) & $(\mathbf{1 0 . 0} \mathbf{c m}, \mathbf{4 . 0} \mathbf{c m})$ \\
\hline \multirow{2}{*}{ Energy line $(\mathrm{keV})$} & Simulated measurement \\
\hline 144 & $1.04 \times 10^{2} \pm 9.94 \%$ \\
186 & $9.86 \times 10^{2} \pm 7.55 \%$ \\
766 & $7.85 \times 10^{-1} \pm 3.92 \%$ \\
1001 & $2.52 \times 10^{0} \pm 2.19 \%$ \\
\hline
\end{tabular}

Table 5. Results of the Gelman-Rubin Method for Test Problem 2

\begin{tabular}{rcccc}
\hline \multicolumn{5}{c}{ DREAM with Gelman-Rubin Method } \\
\hline Test & Source radius & Source bottom & Source top & Transport calculations \\
\hline 1 & $3.96 \pm 0.20$ & $1.51 \pm 0.02$ & $6.17 \pm 0.39$ & 23,121 \\
2 & $3.96 \pm 0.14$ & $1.51 \pm 0.02$ & $6.17 \pm 0.33$ & 12,456 \\
3 & $3.96 \pm 0.18$ & $1.51 \pm 0.02$ & $6.16 \pm 0.37$ & 16,758 \\
4 & $3.96 \pm 0.17$ & $1.51 \pm 0.02$ & $6.16 \pm 0.36$ & 38,313 \\
5 & $3.96 \pm 0.18$ & $1.51 \pm 0.02$ & $6.18 \pm 0.36$ & 24,327 \\
6 & $3.95 \pm 0.18$ & $1.51 \pm 0.02$ & $6.19 \pm 0.38$ & 32,868 \\
7 & $3.94 \pm 0.16$ & $1.51 \pm 0.02$ & $6.19 \pm 0.35$ & 17,802 \\
8 & $3.93 \pm 0.18$ & $1.50 \pm 0.02$ & $6.22 \pm 0.37$ & 22,083 \\
9 & $3.96 \pm 0.17$ & $1.51 \pm 0.02$ & $6.16 \pm 0.39$ & 25,929 \\
10 & $3.97 \pm 0.21$ & $1.51 \pm 0.02$ & $6.15 \pm 0.44$ & \\
\hline & & DREAM without Gelman-Rubin Method & 90,000 \\
\hline Test & Source radius & Source bottom & Source top & Transport calculations \\
\hline 1 & $3.96 \pm 0.17$ & $1.51 \pm 0.02$ & $6.16 \pm 0.37$ & 90,000 \\
2 & $3.96 \pm 0.18$ & $1.51 \pm 0.02$ & $6.16 \pm 0.37$ & 90,000 \\
3 & $3.96 \pm 0.18$ & $1.51 \pm 0.02$ & $6.16 \pm 0.38$ & 90,000 \\
4 & $3.96 \pm 0.18$ & $1.51 \pm 0.02$ & $6.18 \pm 0.38$ & 90,000 \\
5 & $3.97 \pm 0.18$ & $1.51 \pm 0.02$ & $6.15 \pm 0.37$ & 90,000 \\
6 & $3.96 \pm 0.18$ & $1.51 \pm 0.02$ & $6.15 \pm 0.38$ & 90,000 \\
7 & $3.97 \pm 0.18$ & $1.51 \pm 0.02$ & $6.15 \pm 0.37$ & 90,000 \\
8 & $3.96 \pm 0.17$ & $1.51 \pm 0.02$ & $6.17 \pm 0.35$ & 90,000 \\
9 & $3.96 \pm 0.18$ & $1.51 \pm 0.02$ & $6.17 \pm 0.38$ & 90,000 \\
10 & $3.96 \pm 0.17$ & $1.51 \pm 0.02$ & $6.18 \pm 0.37$ &
\end{tabular}




\section{SUMMARY AND CONCLUSIONS}

The DiffeRential Evolution Adaptive Metropolis (DREAM) method has previously been shown to be a powerful tool for solving and quantifying uncertainties in inverse transport problems within Los Alamos National Laboratory's inverse transport analysis tool, INVERSE. While the DREAM method is highly adept at accurate uncertainty quantification, it can require a large number of time-consuming particle transport calculations. Previously, there was no built-in capability in INVERSE to determine the number of calculations required for accurate uncertainty quantification, and the user was forced to choose a maximum number. In this work, we have implemented the Gelman-Rubin convergence metric, a method that compares within-chain to between-chain variances, to automatically detect when the DREAM method has successfully found the solution.

Numerical results have shown that previous estimates for the number of transport calculations required were far too large, and the Gelman-Rubin approach has significantly reduced this number and thus has also reduced the run time for the problem. In a numerical test case in a spherical system, the Gelman-Rubin approach reduced the number of transport calculations used by INVERSE by over $90 \%$, and in a cylindrical system, this approach reduced the number of transport calculations used by INVERSE by $75 \%$. 


\section{REFERENCES}

1. J. A. Vrugt, C. J. F. ter Braak, C. G. H. Diks, B. A. Robinson, J. M. Hymon and D. Higdon, "Accelerating Markov Chain Monte Carlo Simulation by Differential Evolution with SelfAdaptive Randomized Subspace Sampling," Int. J. Nonlin. Sci. Num., 10(3), 273-290 (2009).

2. A. Gelman and D. B. Rubin, "Inference from Iterative Simulation Using Multiple Sequences," Stat. Sci., 7, 457-472.

3. J. Bikowski, J. Van Der Kruk, J. A. Huisman, H. Vereecken, and J. A. Vrugt, "Explicit Consideration of Measurement Uncertainty During Bayesian Inversion of Dispersive GPR Data," $6^{\text {th }}$ International Workshop on Advanced Ground Penetrating Radar, IWAGPR 2011, June 22-24, 2011, Aachen, Germany (2011).

4. E. Laloy and J. A. Vrugt, "High-Dimensional Posterior Exploration of Hydrologic Models Using Multiple-Try DREAM(ZS) and High-Performance Computing," Water Resources Research, 48(1) (2012). 